\title{
Quando le cose sono più brutte delle parole: gli aggettivi corrispondenti a omicidio, suicidio, genocidio
}

Paolo D'Achille

PUBBLICATO: 29 GENNAIO 2021

\section{Quesito:}

Ci sono pervenuti vari quesiti che ci chiedono se è corretto l'aggettivo omicidiario, usato nel linguaggio giuridico, spesso riferito all'intento dell'autore del delitto, o se è meglio sostituirlo con omicida (forma della quale alcuni chiedono quale sia il plurale). Altri chiedono se esistono in italiano il verbo genocidiare, il sostantivo genocida e l'aggettivo genocidiario, che corrisponderebbe al francese génocidiaire. Altri ancora hanno dubbi sulla presenza della $i$, chiedendo se non siano preferibili omicidario e genocidario alle forme citate, come pure suicidario a suicidiario.

\section{Quando le cose sono più brutte delle parole: gli aggettivi corrispondenti a omicidio, suicidio, genocidio}

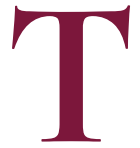

rattiamo di una serie di parole che molti nostri lettori considerano "brutte" e, al riguardo, ci permettiamo subito di notare che, posto che lo siano effettivamente, piu brutte ancora sono le cose a cui si riferiscono: l'uccisione di sé stessi (suicidio), di altre persone (omicidio), perfino di un popolo (genocidio, termine usato peraltro anche in senso figurato: il pensiero va al genocidio dei dialetti di cui parlava Pasolini nel suo ultimo intervento pubblico, Volgar'eloquio). In ogni caso, sebbene siano percepiti dai lettori come neologismi, a parte il verbo genocidiare, tutti gli altri termini da loro segnalati (si tratta sempre di parole dotte o modellate su parole dotte) sono in realtà attestati da tempo (a volte da secoli) in italiano e quasi tutti sono registrati nei principali dizionari, il che, dunque, ne legittima l'uso.

La voce più antica - che è anche, curiosamente, l'unica assente dallo Zingarelli 2O2I - è omicidiario; del resto anche omicidio e omicida, risalenti al sec. XIII, sono vocaboli piu antichi di suicidio e suicida, del sec. XVIII, e di genocidio e genocida, datati I950 nel GRADIT. Il GDLI registra omicidiario sia come nome maschile, equivalente a omicida, e quindi nel senso di 'assassino' e anche di 'sicario', sia come aggettivo, col valore di 'Che spinge, che induce a uccidere; che si alimenta del gusto, del desiderio di uccidere'. In entrambe le funzioni è documentato, al singolare e al plurale, in italiano antico, a partire dal Trecento, come risulta dalla voce del TLIO, che riporta le varie forme attestate (homicidiarij, homicidiario, homicidiariu, omicidiarie, omicidiarii, tra le quali prevalgono quelle con $h$ - per influsso del latino homicidiarius, che ne è alla base) e propone, rispettivamente, le definizioni di 'Chi è direttamente responsabile dell'uccisione di una o più persone. Estens. Individuo capace di crudeltà e atti efferati contro le persone' e di 'Che ha ucciso persone o è capace di farlo, sanguinario'.

La variante omicidario non è registrata nel TLIO sotto la voce appena citata, ma un plurale homicidarii figura (assieme ad altre varianti, al singolare e al plurale, come homecidare, homicidaio, homicidare, mecïaro, mecidaru, michidari, michidaru, miciari, micidari, micidaro, micidiari, micidiaro, omecidaio, omiciaro, omicidaio, omicidare) sotto la voce omicidaio, che parte, comunque, anch'essa dal latino homicidiarius, mentre - per mantenere distinte le sue voci - si potrebbe partire da homicidarius, che è documentato pure in latino (e che forse si può spiegare col fatto che in homicidium la - $i$ semiconsonantica del confisso -cidium è stata considerata esterna al tema, oppure per evitare la sequenza di ben quattro $i$ ). 
Va rilevato, infine, che il termine omicida, diversamente da omicidario e omicidaio, non è attestato in italiano antico come aggettivo (cfr. anche in questo caso la voce del TLIO), il che potrebbe spiegare la ripresa di homicidiarius/homicidarius anzitutto in questa funzione. E cogliamo loccasione per dire (rispondendo a un lettore) che omicida e anche suicida e genocida hanno (sia come nomi, sia come aggettivi) i plurali in $-i$ al maschile e in $-e$ al femminile.

Certo, una volta che omicida ha assunto anche valore aggettivale (il che avviene a fine Quattrocento: i primi esempi citati al proposito dal GDLI e dal DELI sono di Masuccio Salernitano e di Ludovico Ariosto), di omicid(i)ario non c'era più bisogno. Ma l'italiano non disdegna affatto la polimorfia lessicale, e cosiomicidiario, come rilevato giustamente da alcuni lettori, è diventato un termine specialistico del diritto e come tale è registrato nel GRADIT (che segnala anche la variante omicidario), sia come aggettivo ("relativo a omicidio: tasso o[micidiario]"), sia come nome (equivalente a omicida); come nome, tuttavia, ne sconsiglierei senz'altro l'uso.

Non solo, ma proprio sul modello di omicid(i)iario si è formato, molto più recentemente, suicid(i)ario, non lemmatizzato né nel GRADIT né nel GDLI, ma presente nello Zingarelli, di cui riportiamo la voce, dalla quale si coglie il valore esclusivamente aggettivale (la data indicata, 1983, può essere anticipata grazie a Google libri, che fornisce l'esempio di "impulso suicidario" in Aldo Franchini, Simulazione di rapina e strangolamento in un ragazzo sedicenne, "Rivista sperimentale di freniatria e medicina legale", vol.62, I938, p. 342-355: 352):

suicidario /swit $\mathrm{i}^{\mathrm{d} d a r j o /}$ o suicidiario [1983] agg. I di suicidio, relativo a suicidio: rischio sluicidario]; proposito s[uicidario] 2 che conduce al suicidio: pratiche suicidarie

Ancora più tardi, sullo stesso modello derivativo - ma forse anche, come suggerisce un lettore, per influsso del francese génocidaire (non *génocidiaire), passato anche allinglese (ma anche in queste lingue si tratta di neologismi relativamente recenti) - si è formato genocidario, che significa, come aggettivo, 'relativo a genocidio' (Zingarelli) e, come aggettivo o nome, 'che o chi si macchia di genocidio' (Zingarelli e GRADIT). Il termine è datato I986 nello Zingarelli, I997 nel GRADIT che non segnala la variante genocidiario.

Come si diceva all'inizio, invece, il verbo genocidiare non è entrato nei vocabolari e, sebbene abbia alcune occorrenze in rete, più numerose nella forma genocidare (effettivamente preferibile, sul modello di suicidare/suicidarsi), speriamo proprio che non se ne senta mai la mancanza, cosi come ci auguriamo che non si senta mai l'esigenza di formare, sulla base di femminicidio e femminicida, l'aggettivo femminicidario, di cui Google restituisce solo tre occorrenze (ultimo accesso: 22 marzo 2020).

D'altra parte, anche per sdrammatizzare un po', possiamo dire che -cida e -cidio sono stati usati non di rado anche per formazioni occasionali, costruite con nomi comuni (e pure con nomi propri). La produttività di -cida era del resto già stata segnalata, con riferimento al Seicento, da Bruno Migliorini, che parlando "dei numerosi elementi compositivi che già avevano questo valore in latino ma che ora danno largamente origine a parole nuove" afferma:

tra i nomi formati con -cida appaiono e scompaiono coricida (Fioretti), e fioricida (Marucelli), sparirà anche amanticida (Neri), mentre resterà ussoricida (Allegri), in quanto legato a un concetto giuridico; moschicida, foggiato per gioco dal Lalli, tornerà a servire quando si metteranno in commercio dei prodotti moschicidi (Bruno Migliorini, Storia della lingua italiana, Firenze, Sansoni, 1960, p. 487).

Quanto a -cidio, tra i Neologismi. Parole nuove dai giornali (Roma, Istituto della Enciclopedia Italiana, 20o8), Giovanni Adamo e Valeria Della Valle registrano, oltre a femminicidio, anche culturicidio, 
delfinicidio, fratellicidio, sorellicidio e Staticidio. Personalmente, ricordo il cavaliericidio del libretto di Jacopo Ferretti della Cenerentola di Rossini e il nipoticidio prospettato da Paperone nei confronti di Paperino nei fumetti di Topolino che leggevo da ragazzo (e ne trovo oggi isolate attestazioni in rete). Confesso inoltre che, in passato, qualcuno mi ha scherzosamente minacciato di paolicidio o di dachillicidio!

Ebbene una formazione scherzosa terminante in -cidiario sul modello di omicidiario è lemmatizzata nel GDLI (che non registra invece né suicid(i)ario, né genocid(i)ario): si tratta di nuoricidiario 'uccisore di nuore', attestato in una commedia di Jacopo Nelli (sec. XVIII).

Insomma, per concludere, pur con qualche differenza nell'uso o e nel trattamento lessicografico (omicid(i)ario e genocid(i)ario nomi e aggettivi, suicid(i)ario solo aggettivo; di omicid(i)ario si preferisce la forma con la -i-, di suicid(i)ario e genocid(i)ario quella senza; il primo è presente nel GDLI e nel GRADIT, il secondo solo nello Zingarelli, il terzo nel GRADIT e nello Zingarelli, ma non nel GDLI), si tratta di vocaboli ormai accolti, specie come aggettivi, in certi linguaggi settoriali (diritto, giornalismo) e dunque da accettare. Ma nell'uso comune possiamo certamente rinunciare ad adoperarli, usando invece (il meno possibile, si spera) omicida, suicida, genocida anche come aggettivi.

\section{Cita come:}

Paolo D'Achille, Quando le cose sono più brutte delle parole: gli aggettivi corrispondenti a omicidio, suicidio, genocidio , "Italiano digitale", 2021, XVI, 2021/1 (gennaio-marzo)

DOI: $10.35948 / 2532-9006 / 2021.5465$

\section{Copyright 2021 Accademia della Crusca}

Pubblicato con licenza creative commons CC BY-NC-ND 